\title{
Noonan Syndrome 1
}

National Cancer Institute

\section{Source}

National Cancer Institute. Noonan Syndrome 1. NCI Thesaurus. Code C75459.

Noonan syndrome caused by mutations in the PTPN11 gene. 